\title{
Lidil
}

Revue de linguistique et de didactique des langues

$62 \mid 2020$

Recherches actuelles en didactique du lexique : avancées, réflexions, méthodes

\section{Rabot, sécateur, téléphérique : interroger la pertinence des choix lexicaux dans les outils d'évaluation du langage en orthophonie}

Rabot, sécateur, téléphérique: Exploring the Relevance of Lexical Choices in Tools Evaluating Language in Speech-Language Therapy

Christine da Silva-Genest, Loïc Liégeois, Caroline Masson, Christophe Benzitoun et Marine Le Mené

\section{OpenEdition}

Journals

Édition électronique

URL : http://journals.openedition.org/lidil/8422

DOI : 10.4000/lidil.8422

ISSN : 1960-6052

Éditeur

UGA Éditions/Université Grenoble Alpes

Édition imprimée

ISBN : 978-2-37747-226-0

ISSN : $1146-6480$

Référence électronique

Christine da Silva-Genest, Loïc Liégeois, Caroline Masson, Christophe Benzitoun et Marine Le Mené, " Rabot, sécateur, téléphérique : interroger la pertinence des choix lexicaux dans les outils d'évaluation du langage en orthophonie », Lidil [En ligne], 62 | 2020, mis en ligne le 03 novembre 2020, consulté le 05 novembre 2020. URL : http://journals.openedition.org/lidil/8422 ; DOI : https://doi.org/10.4000/lidil. 8422

Ce document a été généré automatiquement le 5 novembre 2020

(C) Lidil 


\title{
Rabot, sécateur, téléphérique:
} interroger la pertinence des choix lexicaux dans les outils d'évaluation du langage en orthophonie

\author{
Rabot, sécateur, téléphérique: Exploring the Relevance of Lexical Choices in \\ Tools Evaluating Language in Speech-Language Therapy
}

Christine da Silva-Genest, Loïc Liégeois, Caroline Masson, Christophe Benzitoun et Marine Le Mené

\section{Introduction}

1 L'acquisition du lexique constitue un champ de recherche largement étudié (Clark, 1993 ; Bassano, Labrell \& Bonnet, 2020) et de nombreux travaux ont notamment mis en évidence des régularités concernant l'âge d'apparition des premiers mots (Kern \& Gayraud, 2010), leur nature grammaticale et sémantique (noms vs verbes) et le rythme d'acquisition, malgré de nombreuses variations rendant toute généralisation particulièrement épineuse (Gopnik \& Choi, 1995 ; Nelson, 1981).

2 Plusieurs facteurs peuvent expliquer ces différences quantitatives et qualitatives, parmi lesquels le statut socioéconomique (Rowe, 2018) ou l'input. L'étude de Huttenlocher, Haight, Bryk, Seltzer et Lyons (1991) a ainsi mis en évidence un lien entre la quantité de données linguistiques reçues par l'enfant entre 14 et 26 mois et son développement lexical ultérieur. En somme, plus l'input est important, plus la taille du lexique l'est également. Toutefois, cette corrélation ne fait pas consensus et le facteur de la diversité lexicale semble jouer un rôle tout aussi important sur le développement lexical (Weizman \& Snow, 2001 ; Huttenlocher, Waterfall, Vasilyeva, Vevea \& Hedges, 2010). 


\subsection{Développement lexical et âge d'acquisition}

3 Les données sur la taille du lexique des enfants sont bien établies jusqu'à 3-4 ans, notamment grâce au questionnaire parental CDI (MacArthur-Bates Communicative Development Inventory; Fenson et coll., 1993), adapté en français sous les formes IFDC (Inventaires français du développement communicatif; Kern \& Gayraud, 2010) et DLPF (Développement du langage de production en français ; Bassano, Labrell \& Bonnet, 2020). Les données sont de plus en plus imprécises à mesure que l'enfant grandit et deviennent impossibles à déterminer finement à l'âge adulte. Certaines études ont montré que le processus d'acquisition lexicale représente une base fondamentale pour la mise en fonctionnement de l'ensemble des compétences linguistiques (Bassano, 2005), en particulier le développement morphosyntaxique (Bates \& Goodman, 2001) et littéracique (David, 2000).

4 En parallèle, des recherches en psycholinguistique explorent aussi l'âge d'acquisition des mots (Bonin et coll., 2003 ; Chalard, Bonin, Meot, Boyer \& Fayol, 2003). Le procédé le plus fréquemment employé pour le mesurer est le test de (re)connaissance du mot à partir d'une tâche de dénomination d'images présentées selon des critères thématiques ou taxonomiques (Hazard, De Cara \& Chanquoy, 2007). Il faut qu'au moins $75 \%$ des enfants d'une tranche d'âge donnée puissent nommer ce qui est représenté sur l'image ainsi que les deux tranches d'âge au-dessus. Toutefois, la sélection de ces images interroge puisqu'elle fait rarement l'objet d'une validation sur des populations d'enfants (Cannard, 2006). On sait en outre qu'un objet peut être catégorisé de différentes façons et qu'une image - parfois polysémique lorsqu'elle est décontextualisée - peut être interprétée de multiples façons.

Le critère de la fréquence entre également en jeu pour mesurer l'acquisition lexicale. En effet, une corrélation positive entre fréquence et âge d'acquisition est observée : plus un mot est fréquent, plus il est produit et acquis tôt par l'enfant (Ambridge, Kidd, Rowland \& Theakston, 2015 ; Kern, 2019).

6 Dans les travaux en pathologie de l'acquisition du langage, les études montrent que les enfants présentant un trouble du développement langagier produisent leurs premiers mots plus tardivement que les enfants à développement typique de même âge chronologique (Hick, Conti-Ramsden, Serratrice \& Faragher, 2002), et ont un vocabulaire plus restreint (McGregor et coll., 2013 ; Rice \& Bode, 1993). Les recherches expérimentales relèvent aussi que ces enfants ont des difficultés à comprendre et à dénommer des items cibles (Rice, Buhr \& Oetting, 1992), à apprendre les caractéristiques sémantiques des nouveaux mots (Mainela-Arnold, Evans \& Coady, 2010) ou encore à les définir (Ponari, Norbury, Rotary, Lenci \& Vigliocco, 2018).

7 Ces difficultés lexico-sémantiques ne sont pas propres aux troubles spécifiques du développement du langage oral. En effet, d'autres populations (syndrome de Down, surdité, retard de langage) présentent des décalages temporels dans l'acquisition lexicale et un stock lexical réduit (Rescorla, Alley \& Christine, 2001 ; Stiles, McGregor \& Bentler, 2012; McGregor, Oleson, Bahnsen \& Duff, 2013; Walker, Redfern \& Oleson, 2019). 


\section{2. Évaluation des capacités lexicales des enfants}

8 Afin d'apprécier les compétences et les difficultés des sujets, le clinicien et/ou chercheur doit avoir à sa disposition des outils d'évaluation adaptés à ses objectifs. Quel que soit le niveau linguistique évalué, les différentes épreuves d'évaluation sont caractérisées selon qu'elles induisent ou non des productions linguistiques particulières (p. ex., répétition de mots vs récit) et qu'elles évaluent les connaissances du sujet sur la langue (épreuves décontextualisées) ou les compétences mobilisées dans un contexte communicationnel spécifique (activités contextualisées). L'utilisation des tests de langage permet de confirmer ou d'infirmer des déficits langagiers et d'apprécier le degré de sévérité d'un trouble, mais ne permet pas de relever les compétences des sujets testés (Rondal, 2003; De Weck \& Marro, 2010). Il est donc nécessaire d'avoir recours à des méthodes complémentaires pour apprécier les compétences linguistiques réelles des sujets.

9 Les outils d'évaluation sont rarement analysés dans la littérature (Rondal, 2003). Pourtant, les cliniciens privilégient les batteries de tests de langage alors qu'elles soulèvent des questions sur la prise en compte ou non des différences socioculturelles et des variantes langagières des sujets ainsi que sur l'évaluation des usages de la langue (Da Silva-Genest \& Masson, 2017). Ainsi, il nous semble primordial de comprendre d'une part, la façon dont les tests de langage sont construits, et d'autre part, les choix effectués par les auteurs. En nous penchant sur l'évaluation lexicale, nous nous interrogerons plus particulièrement sur la pertinence des choix des items lexicaux au sein des épreuves de dénomination dans les tests de langage évaluant les compétences lexico-sémantiques d'enfants âgés entre 4 et 8 ans. Pour ce faire, nous nous proposons d'étudier deux sources de données: d'une part, celles issues des tests de langage et, d'autre part, celles issues de situations d'interactions verbales naturelles nous permettant d'accéder aux productions réelles des enfants. Une comparaison de ces deux types de données sera réalisée dans le but d'observer leurs similitudes et divergences.

\section{Méthode}

d'évaluation des compétences lexico-sémantiques. Nous avons donc établi un inventaire des épreuves et des items lexicaux utilisés dans des tests de langage à destination d'enfants âgés entre 2;03 et 8 ans. Cinq tests de langage ont été sélectionnés : EVALO 2-6 (Coquet, Roustit \& Ferrand, 2009); EXALANG 3-6 (Helloin \& Thibault 2006) ; EXALANG 5-8 (Croteau et coll., 2010); ELO (Khomsi, 2001) et N-EEL (Chevrie-Muller \& Plaza, 2001). Ces tests ont été choisis parce que ce sont les plus utilisés par les orthophonistes en France (comme mentionné notamment dans une étude portant sur les bilans orthophoniques menée par Caët, Ravez, Moutel \& Fragnon, 2019). Pour chaque test de langage, nous avons relevé les épreuves ciblant explicitement le lexique (en réception ou en production) et tous les items associés ont été répertoriés.

12

Dans un deuxième temps, nous avons constitué, à partir de données longitudinales issues de corpus d'interactions parents-enfants en situations naturelles (jeu, repas, 
bain...) (corpus CoLaJE [ 6 enfants, Morgenstern \& Parisse, 2012], ALIPE [3 enfants, Liégeois, Chanier \& Chabanal, 2014] et Lyon [6 enfants, Demuth \& Tremblay, 2008]), une liste de fréquence des formes produites et entendues par quinze enfants âgés de 10 mois à 6 ans dans des situations naturelles. Cet agrégat de corpus représente 2422113 mots, dont 532319 sont produits par les jeunes sujets visés par le recueil de données (tableau 1). Les données ont été regroupées en fonction de la disponibilité des bases en question et ne constitue donc pas un corpus construit en tant que tel. Il n'est pas représentatif notamment du point de vue des caractéristiques socioéconomiques des enfants ou géographique. Les enfants sont tous français, à l'exception d'une qui est belge. Par ailleurs, nous avons sélectionné des productions d'enfants de moins de 2 ans afin d'avoir des données sur l'input et de connaitre la fréquence des termes entendus par les enfants pour apprécier l'âge potentiel d'apparition d'un lexème. Pour mener à bien nos analyses, nous avons utilisé le logiciel TXM (Heiden, Magué \& Pincemin, 2010). Les données ont ensuite été exportées de TXM vers un tableur afin d'être exploitées.

Enfin, dans un troisième et dernier temps, nous avons comparé les résultats obtenus dans les deux premières étapes de l'étude. Cette comparaison a pour but, d'une part, de relever la correspondance ou non des formes et de leur fréquence dans les tests de langage et dans les corpus d'interactions et, d'autre part, de mener une réflexion sur la pertinence ou non des choix d'items lexicaux.

\section{Résultats}

\subsection{Phase 1 : inventaire des épreuves lexico-sémantiques}

Tout d'abord, nous présentons les différentes épreuves proposées par les tests de langage pour évaluer les compétences lexico-sémantiques (3.1.1) puis nous décrivons de façon plus approfondie l'épreuve la plus fréquente, à savoir celle de dénomination (3.1.2).

\subsubsection{Types d'épreuves lexico-sémantiques}

Parmi les cinq tests sélectionnés, nous avons relevé 26 épreuves (tableau 1) permettant de tester les compétences lexico-sémantiques. Parmi ces épreuves, deux formes sont principalement proposées: la désignation d'images (ou d'objets) pour tester la compréhension lexicale et la dénomination pour évaluer la production lexicale.

Tableau 1. - Liste des épreuves testant les compétences lexico-sémantiques.

\begin{tabular}{|l|l|l|l|l|}
\hline EVALO 2-6 & EXALANG 3-6 & EXALANG 5-8 & ELO & N-EEL \\
\hline \multicolumn{5}{|l|}{ Nombre total d'items sélectionnés par les épreuves lexico-sémantiques } \\
\hline 300 & 123 & 45 & 80 & 121 \\
\hline
\end{tabular}




\begin{tabular}{|c|c|c|c|c|}
\hline $\begin{array}{l}\text { Jeu partagé (lexique en } \\
\text { dénomination et } \\
\text { désignation) }\end{array}$ & $\begin{array}{l}\text { Dénomination } \\
\text { d'images }\end{array}$ & Dénomination & $\begin{array}{l}\text { Lexique en } \\
\text { réception }\end{array}$ & $\begin{array}{l}10 \text { A. Expression mots } \\
\text { abstraits } \\
\text { (pour les enfants de plus } \\
\text { de 5;07 ans ; Forme G) }\end{array}$ \\
\hline $\begin{array}{l}\text { Dénomination } \\
\text { phonologie/lexique }\end{array}$ & $\begin{array}{l}\text { Dénomination de } \\
\text { couleurs }\end{array}$ & $\begin{array}{l}\text { Fluence } \\
\text { sémantique }\end{array}$ & $\begin{array}{l}\text { Lexique en } \\
\text { production }\end{array}$ & $\begin{array}{l}11 . \quad \text { Expression } \\
\text { vocabulaire - } \\
\text { dénomination } \\
\text { vocabulaire } 1 \quad \text { "mots } \\
\text { concrets» }\end{array}$ \\
\hline $\begin{array}{l}\text { Désignation à partir } \\
\text { d'un mot }\end{array}$ & $\begin{array}{l}\text { Dénomination } \\
\text { rapide couleurs }\end{array}$ & & & $\begin{array}{l}11 . \quad \text { Expression } \\
\text { vocabulaire - } \\
\text { dénomination } \\
\text { vocabulaire } 2 \text { (couleurs, } \\
\text { formes, parties du } \\
\text { corps) }\end{array}$ \\
\hline $\begin{array}{l}\text { Désignation à partir } \\
\text { d'un indice }^{[1]}\end{array}$ & $\begin{array}{l}\text { Topologie (en } \\
\text { production) }\end{array}$ & & & $\begin{array}{l}\text { 13. Compréhension - } \\
\text { mots abstraits }\end{array}$ \\
\hline $\begin{array}{l}\text { Compréhension de } \\
\text { qualificatifs }^{[1,2]}\end{array}$ & $\begin{array}{l}\text { Quantificateurs } \\
\text { et nombre: } \\
\text { langage } \\
\text { mathématique }\end{array}$ & & & $\begin{array}{l}\text { 16. Compréhension - } \\
\text { Lexique } 1 \text { (mots } \\
\text { concrets) }\end{array}$ \\
\hline $\begin{array}{l}\text { Compréhension de } \\
\text { termes topologiques }\end{array}$ & $\begin{array}{l}\text { Topologie (en } \\
\text { réception) }\end{array}$ & & & $\begin{array}{l}\text { 16. Compréhension - } \\
\text { Lexique } 2 \text { (couleurs, } \\
\text { formes, parties du } \\
\text { corps) }\end{array}$ \\
\hline $\begin{array}{l}\text { Lexique induit }^{[1]} \\
\text { [Tâches diverses : } \\
\text { catégorisation } \\
\text { générique, partie/tout, } \\
\text { fluence sémantique, } \\
\text { évocation à l'aide d'un } \\
\text { indice sémantique, } \\
\text { antonymes, définition, } \\
\text { productions de } \\
\text { qualificatifs à partir } \\
\text { d'une image] }\end{array}$ & $\begin{array}{l}\text { Désignation des } \\
\text { parties du corps }\end{array}$ & & & \\
\hline $\begin{array}{l}\text { Lexique - Mise en } \\
\text { réseaux }\end{array}$ & $\begin{array}{l}\text { Désignation } \\
\text { d'images }\end{array}$ & & & \\
\hline
\end{tabular}

[1] Épreuves présentées dans la version longue seulement (vs version courte).

[2] Épreuves proposées uniquement dans la version « Grands » (4;03 à 6;03 ans). 

présenté par une image (pour les termes concrets) ou d'indiquer à quoi se réfère une notion (pour les termes abstraits). Il s'agit donc d'une activité décontextualisée et métalinguistique (De Weck \& Marro, 2010; Da Silva-Genest, 2017). Une seule activité de dénomination est proposée de façon contextualisée dans le cadre d'une activité de jeu partagé (Forme P d'EVALO 2-6). dénomination constituée de 44 à 85 items pour une même épreuve. Comme pour l'ensemble des épreuves lexico-sémantiques, la classe des noms est la catégorie la plus fréquente ( 257 noms; $75,81 \%$ des items) dans les épreuves de dénomination. À l'inverse, les autres catégories grammaticales sont peu ciblées et seulement par certains tests (ELO et EVALO 2-6 pour la catégorie des verbes en dénomination). apparait dans les cinq tests : chat, qui est également l'un des termes les plus utilisés pour toutes les épreuves évaluant la compétence lexico-sémantique. En effet, il apparait dans 8 épreuves au total. souligné le caractère polysémique, nous nous sommes demandé si, pour chaque image, plusieurs dénominations étaient acceptées (tableau 2). 
Tableau 2. - Nombre de dénominations différentes acceptées dans les épreuves de dénomination.

\begin{tabular}{|l|l|l|l|}
\hline Test de langage & Une seule dénomination & Plusieurs dénominations & Total \\
\hline ELO & 46 & 14 & 60 \\
\hline EVALO 2-6 & 111 & 2 & 113 \\
\hline EXALANG 3-6 & 52 & 9 & 61 \\
\hline EXALANG 5-8 & 44 & 0 & 44 \\
\hline NEEL & 55 & 6 & 61 \\
\hline Total & 308 & 31 & 339 \\
\hline
\end{tabular}

Sur 339 images à dénommer, seules 31 acceptent plusieurs dénominations (soit 9,14\% des items). C'est le cas par exemple de l'item table à repasser pour lequel planche à repasser est accepté ou de crapaud pour grenouille.

L'analyse des dénominations acceptées révèle qu'il peut s'agir de synonymes (se coiffer / se brosser; horloge / pendule), de relations d'hyperonymie ou d'hyponymie (lit / berceau), et de variantes langagières (pince à linge / épingle à linge). Toutefois, très peu de variantes langagières et régionales sont considérées, ce qui souligne une vision normative de la langue.

27 Certaines dénominations acceptées sont liées à une difficulté d'interprétation de l'image. C'est le cas de l'image ci-dessous pour laquelle les dénominations suivantes sont acceptées : tube de dentifrice / dentifrice / colle / pommade / peinture / crème. L'image, proposée hors contexte, est en effet assez difficile à interpréter.

Figure 1. - Item 13 de l'épreuve lexique en production d'ELO (Khomsi, 2001).

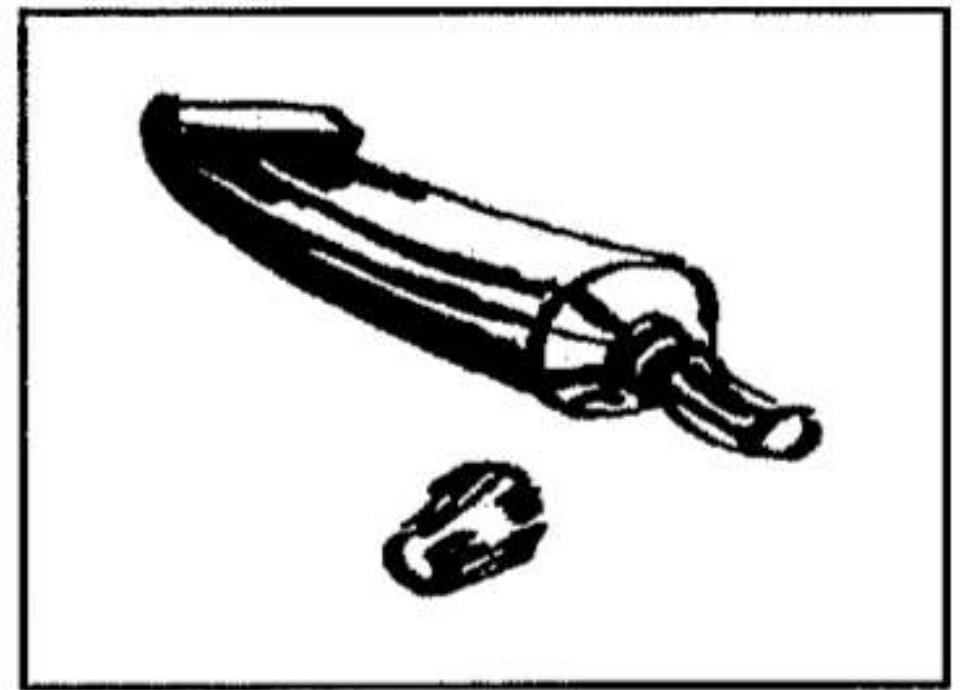




\subsection{Phase 2 : fréquence des formes produites et entendues en situations naturelles d'interaction}

Cette deuxième phase d'analyse, empirique et descriptive, présente le lexique produit et entendu par la quinzaine d'enfants dont les énoncés et ceux des adultes qui les entourent composent notre agrégation de corpus. Alors que 24362 formes différentes sont prononcées dans l'ensemble de l'agrégat, les enfants que nous observons emploient, quant à eux, seulement 9843 formes différentes. Sans surprise, la distribution des fréquences d'usage observées dans notre agrégation est conditionnée par la loi de Zipf (1949), c'est-à-dire que, comme tout corpus linguistique, les transcriptions que nous analysons sont majoritairement composées de formes peu fréquentes : 14978 formes différentes ne sont présentes qu'une ou deux fois dans nos données alors que seulement 1383 formes sont employées 100 fois ou plus. Si on ne se focalise que sur les formes enfantines, plus d'un tiers ne sont produites qu'à une seule reprise, et donc par un seul enfant (soit 3600 hapax).

Pour obtenir les résultats que nous présentons ci-dessous, nous avons pris en compte les quatre paramètres suivants que nous avons appliqués sur les lemmes : la fréquence, l'âge de l'enfant, le nombre d'enfants et l'annotation en catégories grammaticales. Entre 1 an et 2 ans, au moins 10 enfants sur les 12 produisent les lemmes suivants :

- parmi les noms : maman (1156), papa (436), pain (93), ballon (102), eau (233), caca (96), main (44), nez (93), bébé (370) ;

- parmi les verbes : avoir (281), être (827), boire (42), finir (73), aller (85), regarder (262), mettre (197), vouloir (164), attendre (56), pouvoir (18), venir (17), casser (175), ouvrir (65), donner (74), manger (70), voir (59), tomber (104) ;

- parmi les adjectifs : petit (110), rouge (121) ;

- parmi les adverbes : encore (621).

Le tableau ci-dessous présente les lemmes produits par l'ensemble des enfants de 2 à 3 ans.

Tableau 3. - Lemmes produits par tous les enfants de 2 à 3 ans.

\begin{tabular}{|l|l|l|l|l|l|}
\hline \multicolumn{2}{|l|}{ Noms } & \multicolumn{2}{l|}{ Verbes } & Adjectifs & Adverbes \\
\hline maman (3919) & tête (216) & être (11791) & savoir (441) & petit (1308) & encore (1302) \\
\hline papa (1521) & gâteau (200) & avoir (4537) & prendre (345) & autre (716) & aussi (697) \\
\hline bébé (784) & pipi (186) & aller (2578) & finir (336) & gros (426) & ici (596) \\
\hline eau (486) & lit (168) & faire (2250) & donner (243) & rouge (392) & après (294) \\
\hline voiture (405) & bain (155) & vouloir (2231) & partir (234) & bleu (361) & merci (222) \\
\hline main (254) & nez (147) & mettre (1551) & monter (204) & jaune (312) & beaucoup (139) \\
\hline maison (235) & éléphant (137) & voir (903) & fermer (172) & vert (291) & déjà (81) \\
\hline pied (223) & porte (91) & attendre (703) & ranger (163) & beau (183) & \\
\hline
\end{tabular}




\begin{tabular}{|l|l|l|l|l|l|}
\hline & & casser (523) & venir (148) & & \\
\hline & & manger (491) & tomber (616) & & \\
\hline
\end{tabular}

Ci-dessous, nous dressons la liste des noms et des verbes (tableaux 4 et 5) en fonction de leur apparition à un âge donné, c'est-à-dire sans qu'aucune occurrence n'ait été attestée à un âge précédent.

Tableau 4. - Noms apparaissant entre 2 et 5 ans (ordre décroissant).

\begin{tabular}{|l|l|l|l|l|}
\hline \multicolumn{2}{|l|}{ 2-3 ans } & \multicolumn{2}{l|}{ 3-4 ans } & 4-5 ans \\
\hline truc (112) & droit (37) & parcours (28) & ravioli (7) & devinette (9) \\
\hline cache (54) & cœur (35) & costume (24) & contrebasse (6) & immeuble (8) \\
\hline fois (49) & galipette (34) & prénom (22) & accordéon (6) & gratin (8) \\
\hline jambe (48) & poney (32) & sifflet (17) & apéritif (6) & tarama (8) \\
\hline thé (46) & pantoufle (32) & voleur (15) & banc (6) & noyau (7) \\
\hline tigre (46) & tablier (31) & cantine (13) & faon (6) & phrase (5) \\
\hline galop (44) & bougie (31) & flash (12) & mitre (6) & mystère (5) \\
\hline argent (44) & gens (30) & vin (9) & parfum (6) & ambre (5) \\
\hline crotte (41) & jardin (28) & bibendum (9) & serveur (6) & clapier (5) \\
\hline momie (37) & trottinette (26) & pauvre (10) & étage (5) & palet (5) \\
\hline pie (40) & 292 de plus & caramel (8) & 10 de plus & \\
\hline & muscat (8) & & \\
\hline
\end{tabular}

Tableau 5. - Verbes apparaissant entre 2 et 5 ans (ordre décroissant).

\begin{tabular}{|l|l|l|l|}
\hline \multicolumn{2}{|l|}{ 2-3 ans } & 3-4 ans & 4-5 ans \\
\hline croire (65) & remonter (37) & claquer (10) & alterner (15) \\
\hline tromper (53) & oublier (35) & pleuviner (8) & griller (7) \\
\hline nettoyer (49) & réveiller (33) & déborder (8) & bâiller (5) \\
\hline défaire (41) & préférer (28) & agripper (7) & délivrer (5) \\
\hline devoir (41) & prêter (25) & envoyer (6) & soupirer (5) \\
\hline
\end{tabular}




\begin{tabular}{|l|l|l|l|}
\hline changer (40) & rincer (23) & & \\
\hline raconter (40) & 98 de plus & & \\
\hline reculer (38) & & & \\
\hline
\end{tabular}

Nous avons également observé les productions des adultes (les parents des enfants majoritairement). Dans ce cadre, nous nous sommes intéressés aux formes les plus fréquemment utilisées par les adultes et non reprises par les enfants (tableau 6).

Tableau 6. - Formes produites par les adultes et non reprises par les enfants.

\begin{tabular}{|l|l|l|l|}
\hline Noms & Verbes & Adjectifs & Adverbes \\
\hline voyou (48) & proposer (81) & pire (46) & autrement (109) \\
\hline message (47) & savonner (64) & agréable (44) & effectivement (65) \\
\hline température (43) & fonctionner (58) & & apparemment (51) \\
\hline & remarquer (48) & & \\
\hline & prévoir (46) & & \\
\hline
\end{tabular}

Le verbe proposer est utilisé à 81 reprises mais jamais par un enfant. De même, certains adverbes en -ment fréquemment utilisés par des adultes ne le sont jamais par des enfants. Notons également que par rapport à (36), dont (29) et lorsque (27) ne sont pas produits par les enfants, ce qui montre que ces unités sont inexistantes ou du moins extrêmement peu fréquentes avant un certain âge.

Cette phase d'étude nous permet d'obtenir deux informations principales sur le développement lexical. Tout d'abord, il semble possible, à partir d'un agrégat de corpus d'interactions parents-enfants, de dégager un lexique commun à l'ensemble des jeunes sujets observés. Cette observation permet de définir une base de lexique partagé regroupant les items qui, pour une tranche d'âge donnée, pourrait être déterminée comme acquise par l'ensemble des enfants appartenant à celle-ci ; moyennant, bien évidemment, des disparités en termes géographiques et socioéconomiques, d'où l'intérêt de disposer d'un corpus plus varié pour mener un tel travail. Deuxièmement, elle illustre le fait que la seule intuition du chercheur ou du professionnel de santé ne peut pas servir de point de départ satisfaisant pour établir une liste d'items qui seraient disponibles, en production, par tous les enfants d'une tranche d'âge. En effet, certains items, alors qu'ils apparaissent très fréquemment en input, sont pourtant absents des énoncés enfantins que nous avons pu analyser. 


\subsection{Phase 3 : comparaison des items issus des épreuves de tests de langage et des formes réelles produites par les enfants} compétences lexicales des enfants (phase 1) et leurs productions langagières en situations naturelles (phase 2). Pour cela, nous n'avons sélectionné que les items issus des épreuves de production (vs compréhension). Nous nous sommes donc focalisés sur les 248 items différents proposés par les épreuves de dénomination. À l'aide de l'outil TXM, nous avons procédé à l'extraction de tous les lemmes correspondant aux items des tests de langage fondés sur la dénomination. Par exemple, pour l'item boit (EVALO 2-6, «épreuve phonologie/lexique »), nous avons recherché toutes les formes conjuguées du verbe boire (buvait, bu, boirons, etc.) ; pour un item nominal, nous avons extrait toutes les occurrences pluriel et singulier. De plus, concernant les groupes nominaux tels que tube de dentifrice (ELO), nous avons décidé de rechercher, pour chacun d'eux, l'ensemble des formes qui les composent (tube, dentifrice pour l'exemple précédent). Nous avons exclu de notre recherche d'une part, les onomatopées miaou, vroum et ouah qui ne font pas l'objet d'une analyse dans cette étude et, d'autre part, deux items ambigus à savoir jumelle (qui peut désigner un objet ou une personne) et carte (qui peut désigner une carte géographique ou une carte à jouer).

Une fois ces adaptations effectuées, nous obtenons une liste de 236 items sur laquelle établir les fréquences d'usage dans les productions enfantines et parentales. Si la plupart des items nous amènent à effectuer une requête simple à partir du lemme correspondant, trois types d'items nous obligent à adapter nos requêtes dans l'outil TXM. C'est le cas des noms composés (sac à dos), des locutions adverbiales (en-dessous) et des verbes pronominaux (selaver) pour lesquels on tient compte de l'ensemble des pronoms réflexifs et des formes conjuguées possibles.

requêtes renvoient un total de 114461 occurrences, qui correspondent à 232 items sur les 236 items recherchés. Il s'avère en effet que quatre items sont totalement absents des corpus, productions parentales et enfantines comprises : bouilloire, cocotteminute, salière et sécateur. En revanche, si l'on se focalise uniquement sur les énoncés enfantins, nous relevons 11 items supplémentaires absents de nos données : passoire, paupière, sculpter, rame, lanterne, téléphérique, séchoir, table à repasser, horloge, sèchecheveux et évier. Il est intéressant de noter que, pour ces items, la fréquence d'usage en input est également particulièrement faible puisque la plupart d'entre eux sont employés moins de huit fois par les parents, à la seule exception de l'item évier utilisé 15 fois. À l'inverse, nous observons le cas d'un item (cadenas) produit par un des enfants, mais pas par un des locuteurs adultes.

La principale observation que nous pouvons faire à ce stade concerne la grande disparité des fréquences d'usage des items relevés au cours des interactions naturelles parents-enfant. Ainsi, il s'avère que certains items nominaux sont particulièrement fréquents dans les productions enfantines, comme maman (7079 occurrences), papa (3367 occurrences), bébé (1500 occurrences) ou voiture (868 occurrences). À l'opposé, certains items nominaux n'apparaissent qu'une seule fois, comme harpe, louche ou saxophone par exemple. Une observation similaire est réalisable pour les formes verbales (tricoter n'apparait que 3 fois, contre 1297 pour manger) et les mots fonctionnels (en-dessous n'apparait que 59 fois, contre 1232 pour sur). Les termes produits et leur fréquence sont à mettre en relation avec le contexte de production et la 
situation vécue par les locuteurs. Toutefois, les corpus sélectionnés sont représentatifs d'activités du quotidien et donc des usages associés à celles-ci.

Nous notons donc un faible nombre d'items issus des tests de langage apparaissant très fréquemment dans nos corpus (47 items sur 236 ont une fréquence supérieure à 500), tandis qu'un grand nombre affichent des fréquences proportionnellement très faibles (106 items apparaissent moins de 100 fois en corpus). Cette disparité reflète particulièrement bien les distributions de formes régulièrement observées en corpus (Parisse, 2014). Ainsi, nous pouvons penser que la distribution fréquentielle observée aurait été similaire si les items des tests de dénomination avaient été sélectionnés au hasard dans notre agrégation de corpus. La question que nous pouvons toutefois nous poser concerne l'interprétation des résultats des tests par les professionnels qui les utilisent : la (non) réponse d'un patient concernant un item très fréquent a-t-elle la même valeur que celle pour un item particulièrement rare en usage?

\section{Discussion}

Notre objectif de recherche était d'une part d'avoir des connaissances plus approfondies sur les caractéristiques des épreuves évaluant les compétences lexicosémantiques en orthophonie et, d'autre part, d'apprécier la pertinence des items choisis. Pour y parvenir, nous avons mené une étude exploratoire en trois phases décrites ci-dessus.

\subsection{Caractéristiques des épreuves lexico-sémantiques}

41 L'inventaire et la description des épreuves lexico-sémantiques ont montré une certaine diversité dans les épreuves proposées en production comme en compréhension, surtout pour le test EVALO 2-6 qui est, par exemple, le seul à proposer une épreuve de mise en réseau autour du lexème chat permettant d'apprécier davantage l'organisation du lexique. Cette diversité est cependant relative, car les épreuves sont essentiellement centrées sur des tâches de dénomination pour le versant de la production et des tâches de désignation d'images pour le versant de la compréhension. Ces épreuves se définissent par leur caractère décontextualisé et sans objectif communicationnel.

La description des épreuves a également mis en évidence que sur les 669 items relevés, 351 items sont différents et que ceux utilisés pour évaluer le versant de la production sont plus nombreux que ceux du versant compréhension (397 vs 272). En outre, certains items sont plus fréquents au sein des épreuves que d'autres (voiture et chat par exemple). Les termes les plus fréquents présentent des caractéristiques communes : ce sont des mots concrets (vs abstraits), principalement des noms (voiture, chat, coccinelle, etc.) ou des adjectifs de couleur (bleu, vert). Les verbes, comme les autres catégories grammaticales, sont très peu présents. Concernant la concrétude des termes, on observe que la production et/ou la compréhension de mots abstraits sont rarement évaluées. Cela s'explique par le fait qu'il y a encore très peu de données de la littérature sur l'acquisition des mots abstraits par les enfants à développement typique ou atypique (Ponari, Norbury, Rotaru, Lenci \& Vigliocco, 2018) et que les mots abstraits apparaissent tardivement et sont rares dans le discours enfantin à 40 mois (Bassano, Labrell \& Bonnet, 2020). En outre, il est plus difficile de créer un support adapté illustrant un terme abstrait. En revanche, il est bien plus surprenant que la catégorie 
des verbes soit si peu représentée. En effet, la littérature montre à la fois une apparition plus tardive des verbes dans le discours des enfants à développement typique et un usage spécifique des verbes par les enfants présentant des troubles développementaux du langage oral. Cette sous-représentation peut s'expliquer de différentes façons. D'une part, il est plus difficile de représenter un verbe en image qu'un nom, plus transparent. D'autre part, la production des verbes chez les enfants peut s'avérer plus complexe que celle des noms et des adjectifs lorsqu'ils dénotent des caractéristiques physiques. Ces deux constats pointent les limites de l'évaluation dans une activité totalement décontextualisée et la vision réduite du lexique ou des compétences lexicales à un âge donné. Le recours à l'analyse de productions dites spontanées (Masson et coll., à paraitre) permettrait de pallier ces difficultés en appréciant par exemple la diversité lexicale sur l'ensemble des termes produits et en fonction des catégories grammaticales dans une activité langagière particulière (e.g. jeu symbolique, récit d'expériences personnelles). De plus, l'utilisation de ce type de mesure évaluative a l'avantage d'inclure toutes les variantes langagières et de se rapprocher d'un lexique commun à l'ensemble des jeunes enfants observés en situations naturelles.

La comparaison des items issus des épreuves de dénomination et des formes produites par les enfants en situations naturelles a montré une grande disparité dans la fréquence d'usage réelle des items proposés par les tests. Or, elle n'est pas prise en considération dans les tests de langage puisqu'un même nombre de points est accordé pour la production d'un item, qu'il soit fréquent ou rare. La question de la fréquence est donc neutralisée dans la manière de coter.

\subsection{Questions méthodologiques soulevées par l'analyse des tests de langage}

D'un point de vue méthodologique, différents aspects peuvent être discutés.

Premièrement, les manuels justifient rarement les choix opérés quant à la sélection des items lexicaux. Parmi les cinq tests analysés, les auteurs des N-EEL sont les seuls à présenter une véritable réflexion sur la sélection des items constituant les épreuves lexicales. Dans le manuel d'EXALANG 3-6, les items sont considérés comme adaptés pour la réalisation du test car sélectionnés dans le lexique courant des enfants et correspondant aux références culturelles de l'âge ciblé, sans précisions ni justifications scientifiques. Il est toutefois mentionné que pour ce qui concerne la variation culturelle, la notation est laissée à l'appréciation du testeur. Dans le cas de l'ELO, aucune section n'est dédiée aux choix lexicaux, mais l'on apprend toutefois qu'il s'agit d'objets familiers.

Deuxièmement, il n'est pas attendu, dans les grilles de cotation, que les enfants soient capables de dénommer l'intégralité des items proposés pour les classes d'âge ciblées. À titre d'exemple, les items aubergine et bouilloire (cf. épreuves lexique en production, ELO) ne sont dénommés que par, respectivement, $9 \%$ et $16 \%$ des enfants scolarisés en CM2. Cela signifie donc que l'on propose à des enfants de dénommer des items en sachant à l'avance que les chances qu'ils échouent sont particulièrement élevées. On peut alors s'interroger sur la pertinence et l'intérêt de proposer certains items, d'autant plus lorsque le public cible est déjà plus en difficulté que la moyenne de sa classe d'âge. Le fait de proposer des items plus difficiles que d'autres est nécessaire 
dans le cadre d'une évaluation, mais on pourrait envisager de ne tester que les items dénommés ou désignés par au moins $50 \%$ de la population cible ou de limiter le nombre d'items dans la tâche en fonction de la réussite ou de l'échec de l'enfant comme cela est proposé dans certaines épreuves formelles.

Troisièmement, il est difficile de comprendre quelle est la langue de référence sur laquelle les épreuves se basent. En effet, les items sont-ils sélectionnés en fonction de leur fréquence d'apparition à l'oral ou à l'écrit (et plus précisément, quel type d'oral ou d'écrit)? La question se pose par exemple lorsque l'on voit que pour la plupart des items, une seule et unique dénomination est acceptée. On ne peut penser à l'évaluation du langage oral sans tenir compte de la diversité des pratiques langagières et des usages. L'évaluation du langage oral ne peut pas être considérée au travers des caractéristiques propres à l'écrit, tout comme il est indispensable de se détacher de la norme prescrite par les grammaires qui ne reflète qu'en partie la langue orale.

Quatrièmement, il est intéressant de s'interroger également sur la courbe développementale de référence au niveau lexical. En effet, en plus d'une variabilité extrêmement forte d'un enfant à l'autre dans les premiers stades d'acquisition, d'autres études ont montré qu'une distribution normale des compétences lexicales pour un groupe d'âge donné n'est pas toujours représentative de certaines populations (Dollaghan et coll., 1999 ; Qi, Kaiser, Milan \& Hancock, 2006). Leurs résultats montrent en effet qu'en fonction du statut socioéconomique, les lignes développementales des enfants et leurs performances aux épreuves sont différentes. Cette question est très peu abordée par les auteurs des tests qui proposent des grilles de cotation en ne tenant compte que des moyennes générales et en neutralisant la variable socioéconomique.

\subsection{Problématique des méthodes d'évaluation}

L'activité de dénomination est systématiquement proposée dans les tests de langage pour évaluer le lexique des sujets. Même s'il est primordial qu'un sujet sache catégoriser les objets qui l'entourent, cette seule activité permet-elle de rendre compte des capacités lexicales d'un sujet? Quelles sont les informations obtenues à l'aide d'une tâche de dénomination? Ces mêmes questions peuvent être posées pour les épreuves de désignation d'images. Ce type d'épreuves peut seulement relever les items présents dans le vocabulaire de l'enfant testé. Ces épreuves restent peu informatives et ne permettent pas d'obtenir un profil lexical du sujet. Dès lors que l'on considère que les choix sémantiques des locuteurs sont pragmatiquement situés, il est nécessaire de prendre en considération des outils et méthodes alternatives et complémentaires tels que l'analyse du langage spontané. Bien que certains auteurs s'y intéressent, les méthodes doivent être plus souvent questionnées et faire l'objet d'études plus approfondies (Condouris, Meyer \& Tager-Flusberg, 2003 ; Beech \& Harding, 1993/2018).

L'analyse du langage spontané ne peut être mise en place qu'à condition d'avoir des outils adaptés. Ces outils doivent être élaborés à partir de données naturelles et de grands corpus. Les bases de données lexicales sont des supports utilisés et construits à l'heure actuelle dans des perspectives applicatives, en particulier de construction de matériel lexical pour l'orthophonie. Toutefois, celles-ci sont loin d'être homogènes et peuvent être critiquées sur bien des aspects. En premier lieu, celui de leur taille: certaines bases, trop petites, ne permettent pas d'avoir une estimation fiable des mots rares (Zevin \& Seidenberg, 2002). Les choix opérés lors de la création de la base de 
données sont importants également si l'on souhaite s'en servir pour une population spécifique : si elle ne contient que des données adultes, il existe alors un risque que certains mots soient peu voire non connus des enfants. Par ailleurs, les données présenteront également des différences selon qu'elles proviennent de corpus oraux ou écrits. Enfin, il est nécessaire d'adapter les outils d'analyse issus de la recherche scientifique aux besoins et au temps des professionnels de santé et de les mettre à disposition de la communauté. Sans ces adaptations, ces derniers privilégieront toujours les méthodes formelles à l'utilisation des données issues d'interactions spontanées (Da Silva-Genest \& Masson, 2017).

\section{Conclusion}

Le travail présenté ici a permis de mettre en évidence l'importance accordée au lexique pour l'évaluation du langage oral et de remettre en question la pertinence du choix des items sélectionnés pour constituer des épreuves lexicales.

Cette étude exploratoire demande à être poursuivie par l'analyse d'autres tests de langage et l'ajout de nouvelles données naturelles issues des bases de données existantes pour le français, produites par des enfants et des adultes, et complétées par des données d'enfants à développements atypiques. Ce point est au cœur du projet EVALANG («Évaluation des compétences langagières d'enfants à développement typique et atypique en situations naturelles») et en particulier du groupe de travail REFLEX ( Réflexion sur l'évaluation lexicale») au sein desquels s'inscrit l'étude menée ici.

L'objectif final du projet serait de créer une base de données lexicales accessible qui contiendrait l'âge d'apparition des mots en input et output relevés dans les corpus et qui permettrait aux professionnels de santé d'avoir recours à de nouveaux outils d'évaluation, plus fonctionnels et dynamiques.

\section{BIBLIOGRAPHIE}

Ambridge, Ben, KIDD, Evan, Rowland, Caroline F. \& THEAKSTOn, Anna L. (2015). The Ubiquity of Frequency Effects in First Language Acquisition. Journal of Child Language, 42(2), 239-273.

BATES, Elizabeth \& GOODMAN, Judith C. (2001). On the Inseparability of Grammar and the Lexicon: Evidence from Acquisition, Aphasia, and Real-Time Processing. Language and Cognitive Processes, 12(5-6), 507-584.

BASSANO, Dominique. (2005). Production naturelle précoce et acquisition du langage. Lidil, 31, 61-84.

BASSANO, Dominique, LABRELl, Florence \& BONNET, Philippe. (2020). Évaluer les débuts du langage avec le DLPF. Lexique, grammaire et pragmatique. Disponible en ligne sur <https://halshs.archivesouvertes.fr/halshs-02436994> (consulté le 14 février 2020). 
BEECH, John R. \& HARDING, Leonora. (1993/2018). Assessment in Speech and Language Therapy. Londres : Routledge.

Bonin, Patrick, PeEREMAn, Ronald, Malardier, Nathalie, Meot, Alain \& Chalard, Marylène. (2003). A New set of 299 Pictures for Psycholinguistic Studies: French Norms for Name Agreement, Image Agreement, Conceptual Familiarity, Visual Complexity, Image Variability, Age of Acquisition and Naming Latencies. Behavorial Research Methods, Instrument, and Computers, 35(1), 158-167.

CAËT, Stéphanie, RAvez, Sophie, Moutel, Louise \& Fragnon, Sophie. (2019, novembre). Le lexique dans des comptes rendus de bilan de langage oral de l'enfant. Communication présentée à la Journée d'étude « Développement lexical : descriptions, outils, évaluations », Nancy.

CANnARD, Christine, Bonthoux, Françoise, Blaye, Agnès, Scheuner, Nelly, SchreIBER, Anne-Caroline \& TRINQUART, Jacques. (2006). BD2I : normes sur l'identification de 274 images d'objets et leur mise en relation chez l'enfant français de 3 à 8 ans. L'année psychologique, 106, 375-396.

ChAlARD, Marylène, Bonin, Patrick, Meot, Alain, Boyer, Bruno \& FAYol, Michel. (2003). Objective Age-of-Acquisition (AoA) Norms for a Set of 230 Object Names in French: Relationships with Psycholinguistic Variables, the English Data from Morrison et al. (1997), and Naming Latencies. European Journal of Cognitive Psychology, 15(2), 209-245.

Chevrie-Muller, Claude \& PlazA, Monique. (2001). N-EEL : Nouvelles épreuves pour l'examen du langage. Paris : Les Éditions du Centre de psychologie appliquée.

CLARK, Eve V. (1993). The Lexicon in Acquisition. Cambridge : Cambridge University Press. CONDOURIS, Karen, MEYER, Echo \& TAGER-FLuSBERG, Helen. (2003). The Relationship between Standardized Measures of Language and Measures of Spontaneous Speech in Children with Autism. American Journal of Speech-Language Pathology, 12(3), 349-358.

COQUET, Françoise, RouSTIT, Jacques \& FERRAND, Pierre. (2009). EVALO 2-6. Isbergues : Ortho Édition. CROTEAU, Bénédicte, Helloin, Marie-Christel \& THIBAUlT, Marie-Pierre. (2010). EXALANG 5-8 : Batterie informatisée d'examen du langage oral et écrit. Mont-Saint-Aignan : Orthomotus.

DAvid, Jacques. (2000). Le lexique et son acquisition : aspects cognitifs et linguistiques. Le français aujourd'hui, 131, 31-41.

DA SILVA-GENEST, Christine. (2017). Influence des activités sur les pratiques langagières en orthophonie/logopédie. TRANEL, 66, 157-173.

DA SILVA-GENEST, Christine \& MASSON, Caroline. (2017). Apport de la linguistique de corpus à l'étude des situations cliniques : utilisation de ressources écologiques. Studii de lingvistică, 7 , 89-112.

DE WECK, Geneviève \& MARRO, Pascale. (2010). Les troubles du langage chez l'enfant. Description et évaluation. Issy-les-Moulineaux : Elsevier Masson.

Demuth, Katherine \& TRemblay, Annie. (2008). Prosodically-Conditioned Variability in Children's Production of French Determiners. Journal of Child Language, 35, 99-127.

Dollaghan, Christine A., CAmpbell, Thomas F., Paradise, Jack L., Feldman, Heidi M., Janosky, Janine E., PITCAIRN, Dayna N. \& KURS-LASKY, Marcia. (1999). Maternal Education and Measures of Early Speech and Language. Journal of Speech, Language, and Hearing Research, 42(6), 1432-1443.

Fenson, Larry, Dale, Philip S., Reznick, J. S., Thal, Donna J., Bates, Elizabeth, HartunG, J. P., Pethick, Steve \& ReILly, J. S. (1993). The MacArthur Communicative Development Inventories: User's Guide and Technical Manual. San Diego : Singular Publishing Group. 
GoPNIK, Alison \& CHOI, Soonja. (1995). Names, Relational Words, and Cognitive Development in English and Korean Speakers: Nouns Are Not Always Learned before Verbs. Dans M. Tomasello \& W. E. Merriman (dir.), Beyond Names for Things: Young Children's Acquisition of Verbs (p. 63-80). Hillsdale : Lawrence Erlbaum Associates.

HAZARD, Marie-Claire, DE CARA, Bruno \& CHANQUOY, Lucile. (2007). Normes d'âge d'acquisition objectif des mots et recherche de prédicteurs : importance du choix de la base de fréquence lexicale. L'Année psychologique, 107(3), 427-457.

HeIdEN, Serge, MAGUÉ, Jean-Philippe \& Pincemin, Bénédicte. (2010). TXM : Une plateforme logicielle open-source pour la textométrie - conception et développement. Dans S. Bolasco, I. Chiari \& L. Giuliano (dir.), 10th International Conference on the Statistical Analysis of Textual-DataJADT 2010 (vol. 2(3), p. 1021-1032), Rome : Edizioni Universitarie di Lettere Economia Diritto.

Helloin, Marie-Christel \& ThiBAUlt, Marie-Pierre. (2006). EXALANG 3-6 : Batterie informatisée pour l'évaluation du langage oral et langage écrit. Mont-Saint-Aignan : Orthomotus.

Hick, Rachel F., JosePh, Kate L., Conti-RAMSden, Gina, SERRATRICE, Ludovica \& FARAGHER, Brian. (2002). Vocabulary Profiles of Children with Specific Language Impairment. Child Language Teaching and Therapy, 18(2), 165-180.

Huttenlocher, Janelien, Haight, Wendy, BryK, Anthony, Seltzer, Michael \& Lyons, Thomas. (1991). Early Vocabulary Growth: Relation to Language Input and Gender. Developmental Psychology, 27, 236-248.

Huttenlocher, Janelien, Waterfall, Heidi, Vasilyeva, Marina, Vevea, Jack \& Hedges, Larry V. (2010). Sources of Variability in Children's Language Growth. Cognitive Psychology, 61, 343-365.

KERN, Sophie. (2019). Des premiers mots à l'émergence de la grammaire. Dans S. Kern (dir.), Le développement du langage chez le jeune enfant. Théorie, clinique, pratique (p. 85-111). Louvain-laNeuve : De Boeck Supérieur.

KERN, Sophie \& GAYRAUD, Frédérique. (2010). L'IFDC. Grenoble : Les éditions de la Cigale.

KHOMSI, Abdelhamid. (2001). Évaluation du langage oral. Paris : ECPA.

LIÉGEOIS, Loïc, CHANIER, Thierry \& CHABANAL, Damien. (2014). Corpus globaux ALIPE : Interactions parents-enfant annotées pour l'étude de la liaison. Nancy : Ortolang.

MAINELA-ARNolD, Elina, EVANS, Julia L. \& COADY, Jeffry A. (2010). Explaining Lexical Semantic Deficits in Specific Language Impairment: The Role of Phonological Similarity, Phonological Working Memory, and Lexical Competition. Journal of Speech, Language, and Hearing Research, 53(6), 1742-1756.

MASson, Caroline, DA SILVA-GENEST, Christine, CANUT, Emmanuelle \& CAËT, Stéphanie. (À paraitre). Usages des corpus oraux pour l'étude de l'acquisition du français langue maternelle. Dans C. Benzitoun \& M. Rebuschi (dir.), Les corpus en sciences humaines et sociales (p. 15-48). Nancy : PUNÉditions universitaires de Lorraine.

McGregor, Karla K., Oleson, Jacob, BAHnSEN, Alison \& DufF, Dawna. (2013). Children with Developmental Language Impairment Have Vocabulary Deficits Characterized by Limited Breadth and Depth. International Journal of Language and Communication Disorder, 48(3), 307-319.

Morgenstern, Aliyah \& PARISSE, Christophe. (2012). The Paris Corpus. French Language Studies, 22, $7-12$. 
NELSON, Katherine. (1981). Individual Differences in Language Development: Implications for Development and Language. Developmental Psychology, 17(2), 170-187.

PARISSE, Christophe. (2014). Événements langagiers rares et acquisition du langage. Dans F. Neveu, P. Blumenthal, L. Hriba, A. Gerstenberg, J. Meinschaefer \& S. Prévost (dir.), Actes du CMLF $-4^{e}$ Congrès mondial de linguistique française (vol. 8, p. 1551-1562). EDP Sciences. <https://doi.org/ 10.1051/shsconf/20140801339>.

Ponari, Marta, Norbury, Courtenay F., Rotaru, Armand, Lenci, Alessandro \& Vigliocco, Gabriella. (2018). Learning Abstract Words and Concepts: Insights from Developmental Language Disorder. Philosophical Transactions of the Royal Society of London. Series B, Biological sciences, 373(1752). <https://doi.org/10.1098/rstb.2017.0140>.

QI, Cathy H., KAISER, Ann P., Milan, Stephanie \& HANCOCK, Terry. (2006). Language Performance of Low-Income African American and European American Preschool Children on the PPVT-III. Language, Speech, and Hearing Services in Schools, 37(1), 5-16.

Rescorla, Leslie A., Alley, Amie \& Christine, Joanne B. (2001). Word Frequencies in Toddlers' Lexicons. Journal of Speech, Language, and Hearing Research, 44(3), 598-609.

RICE, Mabel \& BODE, John V. (1993). GAPS in the Verb Lexicons of Children with Specific Language Impairment. First Language, 13, 113-131.

Rice, Mabel, Buhr, JoAnn \& OetTing, Janna. (1992). Specific-Language-Impaired Children's Quick Incidental Learning (QUIL) of Words: The Effect of a Pause. Journal of Speech and Hearing Research, $35,1040-1048$.

RoNDAL, Jean-Adolphe (2003). L'évaluation du langage. Bruxelles : Mardaga.

RowE, Meredith L. (2018). Understanding Socioeconomic Differences in Parents' Speech to Children. Child Development Perspectives, 12(2), 122-127.

StiLES, Derek J., McGREGor, Karla K. \& BeNTLER, Ruth A. (2012). Vocabulary and Working Memory in Children Fit with Hearing Aids. Journal of Speech, Language, and Hearing Research, 55(1), 154-167.

WALKER, Elizabeth A., REDFERn, Alexandra \& OLESON, Jacob J. (2019). Linear Mixed-Model Analysis to Examine Longitudinal Trajectories in Vocabulary Depth and Breadth in Children who Are Hard of Hearing. Journal of Speech, Language, and Hearing Research, 62, 525-542.

WeIZMAn, Zehava Oz \& SNOW, Catherine E. (2001). Lexical Output as Related to Children's Vocabulary Acquisition: Effects of Sophisticated Exposure and Support for Meaning. Developmental Psychology, 37(2), 265-279.

ZEVIN, Jason D. \& SEIDENBERG, Mark S. (2002). Age of Acquisition Effects in Word Reading and Other Tasks. Journal of Memory and Language, 47(1), 1-29.

ZIPF, George K. (1949). Human Behavior and the Principle of Least Effort: An Introduction to Human Ecology. Cambridge : Addison-Wesley.

\section{RÉSUMÉS}

Dans le cadre de la détection de pathologies du langage, les compétences linguistiques sont le plus souvent évaluées au travers de tests ancrés dans des situations formelles pour évaluer le langage des patients et poser un diagnostic. Or, on peut s'interroger sur la pertinence du contenu de ces épreuves, proposées pour apprécier un niveau linguistique. Par exemple, pour évaluer les 
compétences lexico-sémantiques en production orale, une épreuve de dénomination d'items est généralement proposée au patient. Mais très peu d'informations sur les caractéristiques de ces items et la façon dont ils ont été choisis sont fournies dans les manuels d'utilisation desdits tests. On peut pourtant s'interroger sur leur représentativité et leur pertinence. Nous présentons une étude exploratoire articulée autour de trois axes : un inventaire des items lexicaux utilisés dans différents tests de langage pour des enfants de 4;06 à 8 ans; une liste de fréquence des formes produites et entendues par les enfants dans des situations naturelles; une comparaison des résultats obtenus lors des deux premières phases de l'étude, visant à relever la correspondance ou non des formes et de leur fréquence dans les tests de langage et dans les corpus d'interactions et à mener une réflexion sur la pertinence ou non des choix d'items lexicaux.

In the context of language pathologies detection, linguistic skills are mostly assessed through standardized language tests used in formal communicative situations to assess patients' language and make a diagnosis. Yet the relevance of these subtests assumed to assess a linguistic level could be questioned. For instance, to assess lexical-semantic skills in oral production, most of the time the patient has to take a test in which he/she has to label a number of items. But only little information about the characteristics of these items and the way they have been selected are mentioned in the handbooks provided with the tests. However, questions can be raised concerning their representativeness and their relevance. The exploratory study presented here is organized around three main points: an inventory of lexical items used in different language tests for children aged 4;06 to 8 years old; a frequency list of lexical forms produced and heard by children in spontaneous situations; a comparison of the results gathered in the first two phases of the study, aiming at observing the degree of matching between the lexical forms used in standardized language tests and interaction corpora as well as the matching of their frequency, in order to carry out a reflection on the choices of lexical items and the relevance of these choices.

\section{INDEX}

Mots-clés : évaluation, orthophonie, acquisition du langage, lexique, corpus

Keywords : assessment, speech-language therapy, language acquisition, lexicon, corpus

\section{AUTEURS}

\section{CHRISTINE DA SILVA-GENEST}

Université de Lorraine, EA 3450 DevAH, UMR 7118 ATILF

LOÏC LIÉGEOIS

Université Paris Diderot - Paris 7, EA 3967 CLILLAC-ARP, UMR 7110 LLF

\section{CAROLINE MASSON}

Université Sorbonne Nouvelle, EA 7345 CLESTHIA, UMR 7118 ATILF

\section{CHRISTOPHE BENZITOUN}

Université de Lorraine, UMR 7118 ATILF

MARINE LE MENÉ

Université de Strasbourg, EA 1339 LiLPa, EA 7345 CLESTHIA 